\title{
FITNESS CONSEQUENCES OF THE TIMING OF METAMORPHOSIS IN A FRESHWATER CRUSTACEAN
}

BU-1543 -M

October, 2000

\author{
Saran Twombly \\ Nancy Tisch
}

Keywords: metamorphosis, life-history variations, fitness, Diaptomus leptopus, freshwater crustaceans.

\begin{abstract}
Metamorphosis is a common life-cycle transition in organisms as diverse as amphibians, insects, fishes and crustaceans, and the timing of this transition often affects an individual's fitness. Here, we manipulated the timing of the metamorphosis in the freshwater copepod, Diaptomus leptopus, and then followed individuals over their entire life cycle to assess the fitness consequences of variation in age and size at metamorphosis. In 3 separate experiments, individuals were raised in different food conditions: low food $(0.2 \mu \mathrm{g} \mathrm{C} / \mathrm{ml})$ switched to high food $(0.7 \mu \mathrm{g} \mathrm{C} / \mathrm{ml})$, or high food switched to low food, at several different larval and juvenile stages. Control individuals were reared on high or low food concentrations over their entire life cycles. For each individual, we measured age and size at metamorphosis and age and size at maturity; for females we also measured total lifetime egg production and calculated a composite fitness measure, $\lambda$. Statistical analyses showed few effects of treatment on age or size at metamorphosis; of these two traits, only age at metamorphosis correlated significantly with age at maturation, suggesting fitness effects. Changes in food conditions during juvenile stages also had no effect on size at maturity, but caused significant differences in age at maturity. Only age at maturity and egg production covaried significantly with $\lambda$. Because egg production was significantly correlated to age at maturity, the fitness differences we observed may be primarily due to variation in developmental rates and age at maturity. These results suggest that natural selection should favor rapid development in field populations, and that larvae should metamorphose (and perhaps mature) at minimum possible sizes. They also suggest that body size plays a different life-history role in these organisms than is recognized in most poikilotherms.
\end{abstract}


Running Head: Fitness consequences of metamorphosis

Fitness consequences of the timing of metamorphosis in a

freshwater crustacean

\author{
Saran Twombly \\ Department of Biological Sciences \\ University of Rhode Island \\ Kingston, Rhode Island 02881 USA \\ twombly@uri.edu \\ and \\ Nancy Tisch \\ Biometrics Department \\ Cornell University \\ Ithaca, New York 14853-7801 \\ nt34@cornell.edu
}


Abstract

Metamorphosis is a common life-cycle transition in organisms as diverse as amphibians, insects, fishes and crustaceans, and the timing of this transition often affects an individual's fitness. Here, we manipulated the timing of metamorphosis in the freshwater copepod, Diaptomus leptopus, and then followed individuals over their entire life cycle to assess the fitness consequences of variation in age and size at metamorphosis. In 3 separate experiments, individuals were raised in different food conditions: low food $(0.2 \mu \mathrm{g} \mathrm{C} / \mathrm{ml})$ switched to high food $(0.7 \mu \mathrm{g} \mathrm{C} / \mathrm{ml})$, or high food switched to low food, at several different larval and juvenile stages. Control individuals were reared on high or low food concentrations over their entire life cycles. For each individual, we measured age and size at metamorphosis and age and size at maturity; for females, we also measured total lifetime egg production and calculated a composite fitness measure, $\lambda$. Statistical analyses showed few effects of treatment on age or size at metamorphosis; of these two traits, only age at metamorphosis correlated significantly with age at maturation, suggesting fitness effects. Changes in food conditions during juvenile stages also had no effect on size at maturity, but caused significant differences in age at maturity. Only age at maturity and egg production covaried significantly with $\lambda$. Because egg production was significantly correlated to age at maturity, the fitness differences we observed may be primarily due to variation in developmental rates and age at maturity. These results suggest that natural selection should favor rapid development in field populations, and that larvae should metamorphose (and perhaps mature) at minimum possible sizes. They also suggest that body size plays a different life-history role in these organisms than is recognized in most poikilotherms. 
Key Words: metamorphosis, life-history variation, fitness, Diaptomus leptopus, freshwater crustaceans

\section{Introduction}

Complex life cycles are characterized by an abrupt ontogenetic change in an individual's morphology, physiology, behavior and ecology that is defined as metamorphosis (e.g.; Wilbur 1980). This transition is common among diverse organisms ranging from amphibians and insects to fish and crustaceans. Within any of these taxa, the age and size at which metamorphosis occurs varies among individuals (e.g., Wilbur and Collins 1973; Semlitsch and Gibbons 1985; Chambers and Leggett 1987; Forrest 1987; Newman 1992; Twombly 1995). Because the timing of metamorphosis is often assumed to affect an individual's fitness, variation in these traits has attracted considerable theoretical and experimental interest (Alford and Harris 1988; Hensley 1993; Leips and Travis 1994; Bradshaw and Johnson 1995; Hentschel 1999; Morey and Reznick 2000).

Age and size at metamorphosis often affect components of fitness directly. These traits influence age and size at maturity in several amphibians (Berven and Gill 1983; Smith 1987; Semlitsch et al. 1988; Skelly and Werner 1990) and insects (Moeur and Istock 1980; Blakley 1981; Hard et al. 1989; Feltmate and Williams 1991; Bradshaw and Holzapfel 1992; Peckarsky et al. 1993; Ball and Baker 1996); because body size at maturity affects clutch sizes or fecundities (females), mating success (males), and longevity (Taylor et al. 1998), and because age at maturity also affects lifetime fecundity, the fitness consequences of metamorphic timing in 
these species are large. Size and age at metamorphosis also affect other fitness components, including survival. Individuals of the teleost Pleuronectes platessa that are small at metamorphosis suffer increased risk of predation by Crangon crangon (van der Veer and Bergman 1987). In several lecithotrophic (non-feeding) marine invertebrates, delay of metamorphosis reduces post-metamorphic growth rates (e.g., Highsmith and Emlet 1986; Qian et al. 1991; Pechenik et al. 1993), development (e.g., Wollacott et al. 1989), survival (e.g., Highsmith and Emlet 1986; Pechenik and Cerulli 1991; Maldonado and Young 1999), or morphology (Wendt 1996). For example, in Bugula neritina that delay metamorphosis, the lophophore (feeding appendage) is smaller than in those individuals that metamorphose earlier, resulting in reduced competitive ability (Wendt 1996). Similarly, delayed metamorphs of Balanus amphitrite are less competitive, and age at metamorphosis ultimately may affect both lifetime survival and reproduction (Pechenik et al. 1993).

Fitness consequences of age or size at metamorphosis are not ubiquitous however. Among marine invertebrates, delayed metamorphosis incurs few costs in organisms with feeding (planktotrophic) larvae unless the larvae are starved (e.g., Pechenik and Eyster 1989; Miller and Hadfield 1990; Pechenik et al. 1996). It seems that feeding larvae that can delay, but still accomplish, metamorphosis without depleted energy stores experience few negative effects (on body size or subsequent growth and survival) of this delay. Fitness consequences of the timing of metamorphosis are also reduced or absent in insects that feed as adults and obtain resources affecting maturation or fecundity after metamorphosis (e.g., Anholt et al. 1991; McPeek 1997). However, Werner (1988) proposed that the effects of age or size at metamorphosis on fitness do not depend solely on the amount of growth accomplished before or after metamorphosis, because 
metamorphosis is still considered an important fitness component in amphibians that accomplish substantial growth during their terrestrial (juvenile) phase.

When the fitness consequences of the timing of metamorphosis are substantial, factors affecting larval growth and development will be important in molding individual life histories. Selection should prolong the larval period or maximize growth during this period when the fitness consequences of body size at metamorphosis are large (Wilbur and Collins 1973; Peckarsky et al. 1993; McPeek and Peckarsky 1998). In contrast, if there are few or no fitness consequences of size at metamorphosis, this transition should be accomplished at the minimum possible size and as early as is developmentally possible (McPeek and Peckarsky 1998).

Environmental conditions, including mortality due to predation, can have profoundly different effects on the evolution of life histories depending on the relative contribution of stage-specific growth and development to individual fitness. Identification of fitness consequences of age and size at metamorphosis is important both to understand how variation in these traits is maintained and to identify selection pressures important in the evolution of life histories among populations and taxa.

Many crustaceans have a metamorphosis as part of their life cycles. For the majority of crustaceans studied, feeding and growth continue throughout the juvenile period and adults also continue to feed but may not grow. Both age and size at metamorphosis are variable in crustaceans (e.g., Hartnoll and Dalley 1981; Twombly 1995; James-Pirri 1996 ) and one or both of these traits responds to environmental conditions during the larval period (Twombly 1996; Hentschel and Emlet in press). The fitness consequences of age or size at metamorphosis (or 
variation in these traits) are unknown in crustaceans, with the exception of Pechenik et al.'s (1993) data showing that older metamorphs of the barnacle, Balanus amphitrite, experience reduced growth which may affect competitive ability and fitness. Copepod crustaceans, for example, experience the majority of their growth after metamorphosis, so that size at metamorphosis may have fewer fitness consequences in these organisms than in many insects or amphibians.

In this study, we quantified fitness consequences of age and size at metamorphosis in the freshwater copepod Dioptomus leptopus. Our previous studies have shown that age and size at metamorphosis vary in this species, and that age is more variable than size (Twombly and Tisch 2000). We suggested that body size throughout the copepod life cycle, including size at metamorphosis, is constrained (Twombly and Tisch 2000). In the following experiments, we manipulated food availability during the larval period to achieve a range of ages and sizes at metamorphosis. We then followed individuals to adulthood to determine the effects of metamorphic timing on size and age at maturity, reproductive output, and a composite fitness measure, $\lambda$. We asked the following questions: 1) how responsive are age and size at metamorphosis to growth conditions during the larval phase, 2) what is the relationship between age and size at metamorphosis and age and size at maturity, lifetime reproductive output and fitness, and 3) What is the relative importance of larval versus juvenile phases in determining individual fitness and therefore the evolution of $D$. leptopus life histories? Based on the growth achieved in this species after metamorphosis, we predicted that age and size at metamorphosis would have few effects on the fitness components we measured. 


\section{Materials and Methods}

General Experimental Design: Diaptomus leptopus ovigerous females were collected from Little Bullhead Pond, Perryville, RI, in June or July 1998 and 1999. Eggs were hatched in the laboratory, when individual nauplii were assigned to 8 experimental treatments - six of these manipulated food concentration (from low to high, or from high to low) during specific developmental stages and 2 served as controls (high food or low food throughout the entire life cycle). All individuals were raised in small petrie dishes in $10 \mathrm{~mL}$ of modified MBL medium (Stemberger 1981 ) at $19^{\circ} \mathrm{C}$ and a $14: 10 \mathrm{~L}: \mathrm{D}$ photoperiod regime. Individuals were examined daily for developmental stage (molting was detected by the presence of exuviae); medium and food were changed every second day. We recorded age and size at metamorphosis for all individuals; size was measured non-destructively using N6 (the last larval stage) exuviae (Twombly and Burns 1996). Following metamorphosis, age and size at all successive juvenile stages were also recorded. Age and size at the molt from C5 to adult were recorded as age and size at maturity. Following maturation, we paired each individual with a non-sibling member of the opposite sex raised on the same food treatment and maintained these pairs in $30 \mathrm{~mL}$ medium at their appropriate food concentration (high or low). Adults were examined every second day, when medium and food were changed. To measure egg production, we isolated females carrying eggs in a small volume of water, briefly anaesthetized them with carbonated water, separated their egg sacs, and returned the females to their culture vessels (Twombly et al. 1998). Eggs were counted after removing the egg sac from the female as eggs often had to be taken out of the egg sac to be counted accurately. A few females produced no eggs, even after being paired with several males. Each experiment ended when individuals died or had stopped producing eggs for 
2 weeks. We used development and egg production data for each surviving female to calculate the composite fitness measure, $\lambda$ (McGraw and Caswell 1996; Twombly et al. 1998).

All copepods were fed a mixture of two algal species, Cryptomonas erosa and Chlamydomonas reinhardtii, that were raised in modified MBL medium (Stemberger 1981) at $19^{\circ} \mathrm{C}$ and a photoperiod regime of $14 \mathrm{hr} \mathrm{L:10} \mathrm{hr} \mathrm{D.} \mathrm{The} \mathrm{concentration} \mathrm{of} \mathrm{stock} \mathrm{cultures} \mathrm{was}$ estimated daily with a hemacytometer and transformed to $\mu \mathrm{g} \mathrm{C} / \mathrm{mL}$ following the equations in Strathmann (1967). Appropriate volumes of stock culture were added to MBL medium to yield $0.10 \mu \mathrm{g} \mathrm{C} / \mathrm{mL}$ of each species for low food conditions (total food concentration $=0.2 \mu \mathrm{g} \mathrm{C} / \mathrm{ml}$ ) or $0.35 \mu \mathrm{g} \mathrm{C} / \mathrm{mL}$ of each species for high food conditions (total concentration $=0.70 \mu \mathrm{g} \mathrm{C} / \mathrm{ml}$ ). Algal cultures were maintained in log phase growth and transferred to new medium weekly.

\section{Experimental Manipulations}

Three different experiments were completed following this general procedure. In the first experiment (June 1998), 15 nauplii from mixed clutches were assigned haphazardly to each of the following 8 treatments: low food or high food over the entire life cycle ( 2 controls designated as $\mathrm{LL}$ or $\mathrm{HH}$ ) and 6 treatments in which food concentration was switched (low to high or high to low) at the third (designated as LHN3 or HLN3), fourth (designated as LHN4 and HLN4), and fifth (designated as LHN5 and HLN5) naupliar (larval) stages. As described above, individuals in all treatments were raised to maturity, mated, and followed until both members of a pair died or until females had stopped producing eggs for 2 weeks. 
Because D. leptopus individuals accomplish approx. $75 \%$ of their growth as juveniles, we began a second experiment (August 1998) to determine the effects of manipulated food concentrations during specific juvenile (copepodite) stages on maturation, egg production, and fitness. As described above, nauplii hatched in the laboratory from field-caught females were reared in batches at high or low food conditions until metamorphosis was achieved. Following metamorphosis 11-20 copepodites were assigned to the following treatments: 2 controls (high and low food for the duration of the life cycle, $\mathrm{HH}$ and LL respectively) and 6 treatments in which food concentration was switched (low to high; high to low) at the second (LHC2, HLC2), fourth (LHC4, HLC4), and fifth (LHC5, HLC5) copepodite stages. Age and size at successive instars were recorded, along with adult parameters described above.

Our third experiment (June 1999) repeated aspects of both previous experiments. Sixty nauplii hatched from field-caught females were assigned to and raised individually in low or high food controls $(\mathrm{LL}, \mathrm{HH})$ or one of 6 treatments in which food concentration was switched (low to high; high to low, as above) at the third naupliar (LHN3, HLN3), first copepodite (LHC1, HLC1) or third (LHC3, HLC3) copepodite stage. We initiated this experiment because overall survival in the laboratory was low (30-70\%) so that sample sizes for fitness analyses from Experiments 1 and 2 were small.

Data Analyses: We used development and reproduction data obtained from individual females to construct age-based matrices (A) to calculate $\lambda$, a composite fitness measure. Each matrix represented a single female, the subdiagonal contained survival probabilities ( 1 for all individuals) and the first row of the matrix contained age-specific fertilities (number of eggs per 
clutch multiplied by 0.5 to account for male contributions to fitness; McGraw and Caswell 1996). The fitness measure, $\lambda$, is the largest real root of the characteristic equation for the matrix A; all matrices were constructed and evaluated using MATLAB software (Mathworks, Natick, $\mathrm{MA})$.

Relationships between age and size at metamorphosis, age and size at maturity (for all individuals), egg production and fitness (females only) were investigated using Spearman Rank correlations (because metamorphosis data were often not normally distributed) followed by a Bonferroni adjustment of $p$ values ( $\alpha=0.05 /$ number of simultaneous correlations calculated). We used Multivariate Analyses of Variance (MANOVA) to examine the contributions of treatment and sex to age and size at metamorphosis and age and size at maturity (all individuals), egg production and fitness (females only) and followed these with univariate ANOVA when significant effects were detected (after Bonferroni correction). When univariate analyses showed significant treatment effects, we used Tukey's HSD post hoc multiple comparison of means test to separate treatment effects. Although metamorphic data were frequently not normally distributed, transformation of these data did not change results of our ANOVA analysis and we performed all ANOVA analyses with untransformed data.

Finally, we used binary logistic regressions followed by $\chi^{2}$ goodness-of-fit tests to determine whether survivorship or reproductive failure (some females survived but failed to reproduce even after multiple matings) was affected by treatment. All statistical tests were run using SAS (SAS Institute 1985) 
Results

Changes in food concentration during several naupliar stages (Experiment 1: N3, N4, N5; Experiment 3: N3) had no effect on either age or size at metamorphosis (non-significant treatment effects, Table 1). Our inability to manipulate the timing of metamorphosis by changing food concentrations during the larval period may well be due to the short overall duration of the naupliar phase.

Although the timing of metamorphosis did not differ among treatments in any experiment, age or size at metamorphosis could still covary with age or size at maturity, indicating fitness effects. Size at metamorphosis did not correlate significantly with size at maturity in Experiment $1(r=0.064, p=0.66, n=50)$ and this correlation was only marginally significant (with Bonferroni correction) in Experiment $3(r=0.22, p=0.005, n=166)$. Correlations between age at metamorphosis and age at maturity were positive and significant (Experiment 1: $\mathrm{r}=0.52, \mathrm{p}=0.0001, \mathrm{n}=50)$ or marginally so (Experiment $3: \mathrm{r}=0.213, \mathrm{p}=0.005, \mathrm{n}=166)$ : the consistent trend in both experiments was that individuals that metamorphosed at an early age also matured early. These correlations indicate that age at metamorphosis (and thus larval development rate) is more likely to contribute to fitness than is size at metamorphosis (or larval growth rates).

Because the naupliar phase accounts for only $20-25 \%$ of the active growth period for $D$. leptopus (and for copepods more generally), we manipulated food concentrations during juvenile stages as well. Size at maturity remained relatively refractory to changes in food conditions 
throughout the juvenile period (Table 1). Changes in food concentration during 3 (Experiment 2) or 2 (Experiment 3) copepodite stages had no effect on size at maturation, although in both experiments, females were larger at maturation than males (significant sex effects; Table 1). In contrast to size at maturity, age at maturity varied significantly with treatment but not with sex (Table 1). In both experiments 2 and 3 , individuals that spent all (HH treatment) or the latter portion of copepodite development at high food concentrations matured earlier than those that spent all (LL treatment) or just a portion of their juvenile phase at low food (Figure 1). As shown above for the timing of metamorphosis, food conditions during the juvenile period primarily affect developmental rates and age at maturity. Only in Experiment 3 were size at maturity and age at maturity significantly correlated $(r=-0.341, p=0.0001, n=166)$.

Figure 1 shows that changes in food to high or low concentrations effected early in copepodite development resulted in an age at maturation equivalent to either the high or the low control individuals. In Experiment 3, individuals that spent the final three copepodite stages at low food matured at the same age as those raised in low food for their entire development, and the same results were obtained for individuals raised on high food (the high food control matured at a similar age as the LHC3 individuals). Although these results demonstrate that copepods respond quickly to changes in food supply, they show that individuals cannot respond immediately (that is, there is some developmental inertia; Bradshaw and Johnson 1995). In Experiment 2 (Figure 1), LHC5 individuals could not catch up with those raised in other high food treatments (HH, LHC2, LHC4) but individuals switched to low food slowed their development rapidly, so that $D$. leptopus responded more quickly to decreased than to increased food concentrations. 
Egg production varied greatly among individuals within a treatment in all experiments (Figure 2), with the result that treatment showed no statistically significantly effect on reproductive output (Table 1). In Experiment 2, individuals initially reared on low food (LL, LHC2, LHC4) produced the largest number of eggs and the largest number of ovigerous females, but this pattern was not repeated in Experiment 3 (1999; Figure 2B).

In all three experiments, changing food concentrations at different stages throughout the life cycle influenced $\lambda$, the composite fitness measure we used (Table 1). In both Experiments 2 and 3, individuals switched from low food to high at all stages except the last copepodite (C5) stage had higher fitness than those switched from high food to low; this trend was particularly clear in Experiment 3 (Figure 3). Size at maturity was unrelated to fitness (Figure 4A; Experiment 1: $r=0.162, p=0.51, n=19$ (not shown in figure); Experiment 2: $r=0.13, p=0.37, n=52$; Experiment 3: $r=0.08, p=0.49, n=84)$ : both small and large-bodied females achieved the same fitness. In contrast, age at maturation clearly varied with fitness: in both Experiments $2(r=-0.76$, $\mathrm{p}=0.0001, \mathrm{n}=52)$ and $3(\mathrm{r}=0.46, \mathrm{p}=0.0001, \mathrm{n}=84)$, the first individuals to mature had the highest fitness (Figure 4B). Total egg production correlated significantly with fitness in Experiments 2 and 3 (Figure 4C: Experiment 2: $r=0.68, p=0.0001, n=52$; Experiment 3: $r=0.79, p=0.0001$, $\mathrm{n}=84$ ). Together, these results indicate that individuals achieved highest fitness by metamorphosing and maturing early or by producing a large number of eggs. In both Experiments 2 and 3, age at maturity correlated significantly with egg production (Experiment 2: $\mathrm{r}=-0.52, \mathrm{p}=0.0001, \mathrm{n}=52 ;$ Experiment $3: \mathrm{r}=-0.36, \mathrm{p}=0.003, \mathrm{n}=84$ ). The significant correlation we found between fecundity and fitness could be due primarily to the covariation of fecundity with age at maturity, providing further support for the importance of developmental rates and age at 
maturity to fitness. Our results also show that factors affecting development during the juvenile phase of the life cycle have the strongest relationship to fitness or its components and suggest that this phase of the life cycle should be most sensitive to selection. Interestingly, in no experiment did body size affect egg production or fitness in $D$. leptopus.

In all experiments and in all treatments, some females survived well but produced no eggs, even when mated with several males. As a result, fitness for these females was 0 , and we eliminated them from our analyses. Binary logistic regression analysis showed no treatment effect on failure to reproduce $\left(\chi^{2}=7.002, p=0.32, \mathrm{df}=6\right)$ so that elimination of these females from our analyses did not bias our ability to detect effects of treatment on fitness or egg production. Survivorship over all experiments ranged from $35-70 \%$; treatment had no significant effect on survival rates (logistic regression $\chi^{2}=7.17, \mathrm{p}=0.31, \mathrm{df}=6$ ). This indicates that none of the developmental stages during which food was manipulated was more sensitive than any other stage. Phenotypic correlations between age and size at metamorphosis in Experiments 1 and 3 were negative but not significant (Experiment 1: $r=-0.24, p=0.10, n=50$; Experiment 3: $r=-0.12$, $p=0.13, n=166)$. At maturity, age and size were significantly correlated only in Experiment $3(r=$ $0.34, \mathrm{p}=0.0001, \mathrm{n}=166)$.

\section{Discussion}

Diaptomus leptopus individuals attain $23-25 \%$ of their total adult body size by the time they reach metamorphosis. Given the post-metamorphic growth this species experiences, we predicted that the timing of metamorphosis would have few effects on the components of fitness 
we measured (age and size at maturation, egg production, and $\lambda$ ). Our results partially confirmed these predictions: body size at metamorphosis was unrelated to size at maturity, and size at maturity had no effect on egg production or fitness. On the one hand, our results agree with those for other organisms that grow substantially after metamorphosis and for which body size at this transition has few significant fitness effects (e.g., Anholt 1991; McPeek 1990, 1997). On the other hand, these results were surprising because body size (at maturation, for example) is traditionally considered an important life history trait (e.g., Roff 1992; Peters 1983). Our results support the conclusion that the fitness consequences of size at metamorphosis depend fundamentally on the organization of an organism's life cycle. They are large when most growth is accomplished before metamorphosis and small or non-existent for organisms that grow as juveniles and adults (see references in McPeek and Peckarsky 1998; Maldonado and Young 1999). While this basic distinction seems to be true most of the time, there are exceptions in the literature: Banks and Thompson (1987) suggested reduced fecundity among individual Coenagrion puella that metamorphosed at a small size, whereas McPeek (1997) and Anholt (1991) report few fitness consequences of size at metamorphosis in damselflies (Enallagma boreale) from the same family .

Given that the larval period in $D$. leptopus accounts for $20-30 \%$ of their total development time, we also predicted that age at metamorphosis should have few fitness effects. This prediction was not confirmed, however. Age at metamorphosis was significantly related to age at maturity in Experiment 1, during which food was manipulated during several larval stages; small differences in the timing of metamorphosis were manifest as differences in maturation. Early metamorphosis corresponded with earlier maturation, which related 
significantly with fitness. The non-significant relationship we found between age at metamorphosis and age at maturity in Experiment 3 may have resulted from the fact that we manipulated food during 1 naupliar stage only in this experiment, resulting in few opportunities for variation in the age at metamorphosis to translate into differences in age at maturity.

While the fitness consequences of body size in organisms such as amphibians and insects are usually straightforward, because larger size confers greater reproductive success, documented consequences of variable ages at metamorphosis are more diverse. Pechenik et al. (1993) and Wendt (1996) showed, for two marine invertebrates that delayed metamorphosis, lowered juvenile growth rates, and suggested that this may lead to reduced fitness. More direct effects on fitness include reduced post-metamorphic survival as metamorphosis is delayed in lecithotrophic larvae of the polychaete Capitella sp. 1 (Pechenik and Cerulli 1991) and the demosponge Sigmadocia caerulea (Maldonado and Young 1999). Negative correlations between development time (specifically, age at metamorphosis) and fitness have been demonstrated for Aedes triseriatus (Hard et al. 1989) and for adult females of the damselfly Enallagma boreale (Anholt 1991), and both Semlitsch et al. (1988) and Smith (1987) showed that age at metamorphosis directly affects age and size at first reproduction in amphibians. Alternatively, no fitness consequences of delayed metamorphosis were detected in planktotrophic marine invertebrates unless larvae were starved (Pechenik et al. 1996). In fact, in diverse species with actively feeding larvae, delayed metamorphosis may have positive effects: mosquitoes (Moeur and Istock 1980), tadpoles (e.g., Wilbur and Collins 1973) and mayflies (e.g., Peckarsky et al. 1993) become larger, and larger sizes confer fitness advantages. In $D$. leptopus, delaying metamorphosis has no fitness advantages because individuals do not grow larger as larval 
duration is prolonged (Twombly and Tisch 2000). Our results agree with those studies showing that younger metamorphs have higher fitness because they mature earlier (e.g., Semlitsch et al. 1988) and indicate that natural selection should favor rapid larval development in field populations.

Taken together, our results suggest that selection in field populations for growth rate or body size (of larvae or adults) should be weak, and that larvae should metamorphose at the minimum possible size. Concurrently, selection should favor rapid larval development, so that individual $D$. leptopus should metamorphose at a minimum size and as soon as developmentally possible. These suggestions match closely the predictions developed by McPeek and Peckarsky (1998) based on the degree to which size at metamorphosis affects adult fecundity. These authors used demographic models to contrast factors affecting fitness in two aquatic insects. In damselflies, size at emergence has little effect on adult fecundity (McPeek 1990, 1997), and fitness (measured as population level $\lambda$ ) is most susceptible to changes in larval mortality. In contrast, in mayflies of the genus Baetis, size at emergence directly affects fecundity (Peckarsky et al. 1993) and fitness varied primarily with larval growth rate rather than with mortality. Their predictions that 1) the timing of metamorphosis in Baetis should vary with environmental conditions while 2) individuals in different damselfly (Enallagma boreale) populations should metamorphosis at the same, minimum body size were confirmed by field studies. Diaptomus leptopus most closely resembles McPeek's damselflies, suggesting that selection should act to shorten the larval period. 
Field studies are needed to identify the effects of body size on mortality in D. leptopus. Substantial research documents the size selectivity of aquatic predators (e.g., Kerfoot and Sih 1987); although body size does not affect reproductive components of fitness (as demonstrated in our laboratory study), it likely affects survival probabilities. In addition, mortality regimes vary among habitats. For example, we collected D. leptopus from a temporary pond (Little Bullhead Pond, Perryville, RI) that is dominated by invertebrate predators. In this habitat, smaller individuals will suffer the highest mortality and selection will favor larger individuals. The opposite sort of pattern would be expected in ponds with vertebrate predators. Inter-habitat differences in size-selective predation likely account for large differences in D. leptopus body sizes among populations (E. Maly, personal communication) and may play a larger role in interpopulational size variation than in maintaining size variation within any particular population.

Our results also show that developmental rates can vary independently from growth. In many studies of metamorphosis, and of life-histories more generally, the relationship between growth and development (are they interdependent or independent processes?) has been questioned. This question is usually answered using phenotypic correlations; the degree to which age and size at metamorphosis are positively, negatively, or un correlated indicates the ways in which these two fundamental processes are coupled (e.g., Wilbur and Collins 1973; Leips and Travis 1994). However Stearns and Koella (1986) argued that, to the extent that growth and development are plastic so that age and size at any transition vary with experimental conditions, phenotypic correlations are misleading. Neither in this study nor in previous research (e.g., Twombly et al. 1998) have we found consistent patterns in phenotypic correlations between age 
and size (at either metamorphosis or maturity), highlighting difficulties in interpreting phenotypic correlations (e.g., Reznick 1985). The experiments we describe here give a clearer indication that development can vary independently of growth and suggest that growth and development are uncoupled in D. leptopus.

Our finding that size at maturation did not correlate with egg production or fitness was surprising with respect to the importance given to body size, particularly in poikilotherms (e.g., Peters 1983; Forrest 1987 and references therein), but extends our previous findings that body size appears constrained in this species (Twombly and Tisch 2000). Regardless of whether a regulatory mechanism acts to constrain body size or not (D. Lytle, personal communication), size at metamorphosis and at maturity vary little in this and other copepod species (Twombly 1994; Twombly and Tisch 2000) and there are no obvious reproductive advantages of being large. In marine copepods, fecundity is influenced more by food quality or nutritional content (of either phytoplankton or zooplankton; Peterson 1985; Peterson and Bellantoni 1987; Peterson et al. 1991; Durbin et al. 1992) than it is by female body size. Limited advantages of body size are not universal among crustaceans, however; larger females produce more eggs in Daphnia and amphipods as well as in several decapod species (e.g., Lynch 1980; Wenner and Kuris 1991). The relationship between female body size and egg number may depend partly on whether eggs are brooded internally for all or a portion of embryonic development.

Patterns of growth observed in D. leptopus represent copepod life cycles more generally; that is, limited growth occurs during a relatively short larval period (which may be designed for dispersal) and the majority of growth occurs post-metamorphosis. The small or negligible fitness 
consequences of the timing of metamorphosis that we document for this species may therefore be widespread in copepods. Data from a second copepod species, Boeckella triarticulata, raised in the laboratory on diets that differed in food quality (Twombly and Burns 1996) showed a significant relationship between age at metamorphosis and age at maturity (Spearman rank correlation coefficient $r=0.469, \mathrm{p}=0.0001, \mathrm{n}=94$ ), but not between size at metamorphosis and size at maturity $(r=-0.086, p=0.41, n=94)$. As reported here for $D$. leptopus, age-based $\lambda$ estimates and age at first reproduction were negatively correlated $(r=-0.692, p=0.0001)$; in addition, the correlation between total reproductive output and $\lambda$ was positive $(r=0.603, p=0.0002)$ (Twombly et al. 1998). These results lend support to a more general conclusion that age at metamorphosis, but not body size, affects fitness in copepods.

The next, important step in building an understanding of how life histories evolve in these numerous and ecologically-important organisms is to determine how predation and other environmental conditions (food quality, for example) affect stage-specific mortality in field populations. The research we have described here provides the foundation for our predictions that, among various field populations, selection will act to minimize the larval period and that differences in body size at metamorphosis will reflect selection on the length of the larval period.

\section{Acknowledgements}

We thank Bob Bullock for the use of his stereomicroscope, Sarah Davidson for help with our experiments, Dina Proestou and Lauren Egee for reading preliminary drafts of the 
manuscript, and the Department of Biological Sciences at University of Rhode Island for financial support.

\section{Literature Cited}

Alford, R.A. and R.N. Harris 1988. Effects of larval growth history on anuran metamorphosis. American Naturalist 131:91-106.

Anholt, B.R. 1991. Measuring selection on a population of damselflies with a manipulated phenotype. Evolution 45:1091-1106.

Anholt, B.R., J.H. Marden and D.M. Jenkins. 1991. Patterns of mass gain and sexual dimorphosm in adult dragonflies (Insecta: Odonata). Canadian Journal of Zoology 69: 1156-1163.

Ball, S.L. and R.L. Baker. 1996. Predator-induced life history changes: antipredator behavior costs or facultative life-history shifts? Ecology 77:1116-1124.

Banks, M.J. and D.J. Thompson. 1987. Regulation of damselfly populations: the effects of larval density on larval survival, development rate and size in the field. Freshwater Biology 17: $357-365$.

Berven, K.E. and D.E. Gill. 1983. Interpreting geographical variation in life-history traits. American Zoologist 23:85-97.

Blakley, N. 1981. Life history significance of size-triggered metamorphosis in milkweed bugs (Oncopeltus). Ecology 62:57-64. 
Bradshaw, W.E. and C.M. Holzapfel. 1992. Reproductive consequences of density-dependent size variation in the pitcherplant mosquito Wyeomyia smithii (Diptera: Culicidae). Annals of the Entomological Society of America 85:274-281.

Bradshaw, W.E. and K. Johnson. 1995. Initiation of metamorphosis in the pitcher-plant mosquito: effects of larval growth history. Ecology 76:2055-2065.

Chambers, R.C. and W.C. Leggett. 1987. Size and age at metamorphosis in marine fishes: an analysis of laboratory-reared winter flounder (Pseudopleuronectes americanuis) with a review of variation in other species. Canadian Journal of Fisheries and Aquatic Science 44:1936-1947.

Durbin, E.G., A.G. Durbin and R.G. Campbell. 1992. Body size and egg production in the marine copepod Acartia hudsonica during a winter-spring diatom bloom in Narragansett Bay. Limnology and Oceanography 37:342-360.

Feltmate, B.W. D.D. Williams. 1991. Evaluation of predator-induced stress on field populations of stoneflies (Plecoptera). Ecology 72:1800-1806.

Forrest, T.G. 1987. Insect size tactics and developmental strategies. Oecologia 73:178-184.

Hard, J.J., W.E. Bradshaw and D.J. Malarkey. 1989. Resource- and density-dependent development in tree-hole mosquitoes. Oikos 54:137-144.

Hartnoll, R.G. and R. Dalley. 1981. The control of size variation within instars of a crustacean. Journal of Experimental Marine Biology and Ecology 53:235-239.

Hensley, F.R. 1993. Ontogenetic loss of phenotypic plasticity of age at metamorphosis in tadpoles. Ecology 74:2405-2412. 
Hentschel, B.T. 1999. Complex life cycles in a variable environment: predicting when the timing of metamorphosis shifts from resource dependent to developmentally fixed. American Naturalist 154:549-558.

Hentschel, B.T. and R.B. Emlet. 2000. Metamorphosis of barnacle nauplii: effects of food variability and a comparison with amphibian models. Ecology, in press.

Highsmith, R.C. and R.B. Emlet. 1986. Delayed metamorphosis: effect on growth and survival of juvenile sand dollars (Echinoidea: Clypeasteroida). Bulletin of Marine Science 39: $347-361$.

James-Pirri, M.-J. 1996. Growth and behavior during the settlement period of the American lobster, Homarus americanus. Ph.D. Dissertation, University of Rhode Island, Kingston, RI, USA.

Kerfoot, W.C. and A. Sih, eds. 1987. Predation: Direct and indirect impacts on aquatic communities. University Press of New England, Hanover, N.H., USA.

Leips, J. and J. Travis. 1994. Metamorphic responses to changing food levels in two species of hylid frogs. Ecology 75:1345-1356.

Lynch, M. 1980. The evolution of cladoceran life histories. Quarterly Review of Biology 55:2342.

Maldonado, M. and C.M. Young. 1999. Effects of the duration of larval life on postlarval stages of the demosponge Sigmadocia caerulea. Journal of Experimental Marine Biology and Ecology 232:9-21.

McGraw, J.B. and H. Caswell. 1996. Estimation of individual fitness from life-history data. American Naturalist 147:47-64. 
McPeek, M.A. 1990. Determination of species composition in the Enallagma damselfly assemblages of permanent lakes. Ecology 71:1714-1726.

McPeek, M.A. 1997. Measuring phenotypic selection on an adaptation: lamellae of damselflies experiencing dragonfly predation. Evolution 51:459-466.

McPeek, M.A. and B.L. Peckarsky. 1998. Life histories and the strengths of species interactions: combining mortality, growth, and fecundity effects. Ecology 79:867-879.

Miller, S.E. and M.G. Hadfield. 1990. Developmental arrest during larval life and life-span extension in a marine mollusk. Science 248:356-358.

Moeur, J.E. and C.A. Istock. 1980. Ecology and evolution of the pitcher-plant mosquito. IV. Larval influence over adult reproductive performance and longevity. Journal of Animal Ecology 49:775-792.

Morey, S. and D. Reznick. 2000. A comparative analysis of plasticity in larval development in three species of spadefoot toads. Ecology 81:1736-1749.

Newman, R.A. 1992. Adaptive plasticity in amphibian metamorphosis. BioScience 42:671-678.

Pechenik, J.A. and T.R. Cerulli. 1991. Influence of delayed metamorphosis on survival, growth, and reproduction of the marine polychaete Capitella sp. I. Journal of Experimental Marine Biology and Ecology 151:17-27.

Pechenik, J.A. and L.S. Eyster. 1989. Influence of delayed metamorphosis on the growth and metabolism of young Crepidula fornicata (Gastropoda) juveniles. Biological Bulletin 176:14-24.

Pechenik, J.A., D. Rittschof and A.R. Schmidt. 1993. Influence of delayed metamorphosis on survival and growth of juvenile barnacles Balanus amphitrite. Marine Biology 115:287294. 
Pechenik, J.A., K. Hammer and C. Weise. 1996. The effect of starvation on acquisition of competence and post-metamorphic performance in the marine prosobranch gastropod Crepidula fornicata (L.). J. exp. Mar. Biol. Ecol. 199:137-152.

Peckarsky, B.L., C.A. Cowan, M.S. Penton and C. Anderson. 1993. Sublethal consequences of stream-dwelling predatory stoneflies on mayfly growth and fecundity. Ecology 74:18361846.

Peters, R.H. 1983. The ecological implications of body size. Cambridge University Press, New York.

Peterson, W.T. 1985. Abundance, age structure and in situ egg production rates of the copepod Temora longicornis in Long Island Sound, New York. Bulletin of Marine Science 37:726-738.

Peterson, W.T. and D.C. Bellantoni. 1987. Relationships between water-column stratification, phytoplankton cell size and copepod fecundity in Long Island Sound and off central Chile. South African Jornal of Marine Science 5:411-421.

Peterson, W.T., P. Tiselius and T. Kiorboe. 1991. Copepod egg production, moulting and growth rates, and secondary production, in the Skagerrak in August 1988. Journal of Plankton Research 13:131-154.

Qian, P-Y. L.R. McEdward and F-S. Chia. 1990. Effects of delayed settlement on survival, growth, and reproduction in the spionid polychaete, Polydora ligni. Invertebrate Reproduction and Development 18:147-152.

Reznick, D. 1985. Costs of reproduction: an evaluation of the empirical evidence. Oikos 44:257267. 
Roff, D.A. 1992. The evolution of life histories: theory and analysis. Chapman and Hall: London.

SAS Institute. 1985. SAS user's guide: statistics. Fifth edition. SAS Institute, Cary, North Carolina, USA.

Semlitsch, R.D. and J.W. Gibbons. 1985. Phenotypic variation in metamorphosis and paedomorphosis in the salamander Ambystoma talpoideum. Ecology 66:1123-1130.

Semlitsch, R.D., D. E. Scott and J.H.K Pechmann. 1988. Time and size at metamorphosis related to adult fitness in Ambystoma talpoideum. Ecology 69:184-192.

Skelly, D.K. and E.E. Werner. 1990. Behavioral and life-historical responses of larval American toads to an odonate predator. Ecology 71:2313-2322.

Smith, D.C. 1987. Adult recruitment in chorus frogs: effects of size and date of metamorphosis. Ecology 68:344-350.

Stearns, S.C. and J.C. Koella. 1986. The evolution of phenotypic plasticity in life-history traits: predictions of reaction norms for age and size at maturity. Evolution 40:893-913.

Stemberger, R.S. 1981. A general approach to the culture of planktonic rotifers. Canadian Journal of Fisheries and Aquatic Sciences 38:721-724.

Strathmann, R.R. 1967. Estimating the organic carbon content of phytoplankton from cell volume or plasma volume. Limnology and Oceanography 12:411-418.

Taylor, B.W., C.R. Anderson and B.L. Peckarsky. 1998. Effects of size at metamorphosis on stonefly fecundity, longevity, and reproductive success. Oecologia 114:494-502.

Twombly, S. 1995. Phenotypic variation in metamorphosis in four species of freshwater copepods. Freshwater Biology 34:29-38. 
Twombly, S. 1996. Timing of metamorphosis in a freshwater crustacean: comparison with anuran models. Ecology 77:1855-1866.

Twombly, S. and C.W. Burns. 1996. Exuvium analysis: a nondestructive method of analyzing copepod growth and development. Limnology and Oceanography 41:1324-1329.

Twombly, S., N. Clancy and C.W. Burns. 1998. Life history consequences of food quality in the freshwater copepod Boeckella triarticulata. Ecology 79:1711-1724.

Twombly, S. and N. Tisch. 2000. Body size regulation in copepod crustaceans. Oecologia 122: 318-326.

Van der Veer, H.W. and M.J.N. Bergman. 1987. Predation by crustaceans on a newly settled 0gropu plaice Pleuronectes platessa population in the western Wadden Sea. Marine Ecology Progress Series 35:203-215.

Wendt, D.E. 1996. Effect of larval swimming duration on success of metamorphosis and size of the ancestrular lophophore in Bugula neritina (Bryozoa). Biological Bulletin 191:224233.

Wenner, A.M. and A.M. Kuris, editors. 1991. Crustacean egg production. Crustacean Issues. Volume 7. A.A. Balkema, Boston, Massachusetts, USA.

Werner, E.E. 1988. Size, scaling and the evolution of complex life cycles. Pages 60-81 in B. Ebenmann and L. Persson (eds.). Size-structured populations. Springer, New York. Wilbur, H.M. 1980. Complex life cycles. Ann. Rev. Ecol. Syst. 11:67-93.

Wilbur, H.M. and J.P. Collins. 1973. Ecological aspects of amphibian metamorphosis. Science 182: $1305-1314$ 
Wollacott, R.M., J.A. Pechenik and K.M. Imbalzano. 1989. Effects of duration of larval swimming period on early colony development in Bugula stonolifera (Bryozoa: Cheilostomata). Marine Biology 102:57-63. 
Table 1. Results of multi- and univariate ANOVA for the effects of treatment and sex on age and size at metamorphosis, age and size at maturity, egg production and fitness for A. Experiment 1 (food changed during 3 larval stages), B. Experiment 2 (food changed during 3 juvenile stages) and $\mathrm{C}$. Experiment 3 (food changed during 1 larval and 2 juvenile stages). Egg production and fitness analyses were not performed for Experiment 1 due to small sample sizes (no replicates for some treatments).

\section{A. Experiment 1 \\ MANOVA test criteria:}

$\begin{array}{llllll} & \text { Wilks' Lambda } & \text { F } & \text { Num DF } & \text { Den DF } & \text { P } \\ \text { Treatment } & 0.4665 & 0.952 & 28 & 113.9 & 0.54 \\ \text { Sex } & 0.351 & 14.33 & 4 & 31 & 0.0001\end{array}$

Univariate Analyses:

$\begin{array}{lllll}\text { Source } & \text { DF } & \text { Type III SS } & \text { F } & \text { P }\end{array}$

Age at Metamorphosis:

$\begin{array}{lllll}\text { Treatment } & 7 & 16.5157 & 2.53 & 0.033 \\ \text { Sex } & 1 & 0.0256 & 0.03 & 0.87\end{array}$

Size at Metamorphosis:

Treatment

7

Sex

1

Age at Maturity: 
Sex

1

7.519

0.86

0.36

Size at Maturity:

$\begin{array}{lllll}\text { Treatment } & 7 & 7773.9 & 0.62 & 0.74 \\ \text { Sex } & 1 & 104737.75 & 58.02 & 0.0001\end{array}$

B. Experiment 2

MANOVA test criteria:

$\begin{array}{lllccc} & \text { Wilks' Lambda } & \text { F } & \text { Num DF } & \text { Den DF } & \text { P } \\ \text { Treatment } & 0.1475 & 9.32 & 28 & 372.8 & 0.0001 \\ \text { Sex } & 0.7496 & 3.99 & 8 & 206 & 0.0002\end{array}$

Univariate Analyses:

Source

DF

Type III SS

F

$\mathbf{P}$

Age at Maturity:

Treatment

7

1004.486

21.41

0.0001

Sex

1

10.635

0.74

0.48

Size at Maturity:

Treatment

7

76792.37

1.86

0.08

Sex

$1 \quad 161157.68$

13.65

0.0001

Egg Production (Females only):

Treatment

7

149436.72

1.73

0.13

Fitness (Females only):

Treatment

$7 \quad 0.0171$

7.53

0.0001 


\section{Experiment 3}

MANOVA test criteria:

$$
\text { Wilks' Lambda F Num DF Den DF P }
$$

Treatment

$$
0.4259
$$

$5.07 \quad 28$

531.43

0.0001

Sex

0.7456

$12.54 \quad 4$

147

0.0001

Univariate Analyses:

Source

DF

Age at Metamorphosis:

Treatment

7

Sex

1

Size at Metamorphosis

Treatment

7

1

$\operatorname{Sex}$

Age at Maturity

Treatment

7

1

Sex

Size at Maturity:

Treatment

7

Sex

Type II SS

F

P 


\section{Figure Captions}

Figure 1. Box plots for age at maturation for Diaptomus leptopus raised under different food conditions. A. Results from Experiment 2, in which food concentrations were switched from low to high $(\mathrm{LH})$ or high to low $(\mathrm{HL})$ and the second (C2), fourth (C4) and fifth (C5) copepodite stages. B. Results from Experiment 3, in which food concentrations were switched from low to high $(\mathrm{LH})$ or high to low (HL) at the first (C1) and third (C3) copepodite stages. Also shown for both experiments are ages at maturation for low food (LL) and high food $(\mathrm{HH})$ controls. The lower boundary of each box indicates the $25^{\text {th }}$ percentile, the line within the box markes the median value ( $50^{\text {th }}$ percentile), and the upper boundary of each box indicates the $75^{\text {th }}$ percentile. Vertical bars show all data points that fall within $\pm 1.5 *$ interquartile range $(\mathrm{IQR})$. Asterisks denote outliers defined as $<1.5^{*} \mathrm{IQR}>$. Similar upper case letters above each boxpolot represent means that are not significantly different as identified by Tukey's HSD post hoc multiple comparisons of means test. Numbers within each box represent the sample size of each treatment.

Figure 2. Box plots for mean total egg production for D. leptopus females raised under different food regimes. A. Results from Experiment 2, in which food concentrations were switched as described in Figure 1. B. Results from Experiment 3 in which food concentrations were switched, as described in Figure 1, for the third naupliar stage (N3) as well as for 2 copepodite stages. Also shown for all experiments are mean estimates for two controls, LL and $\mathrm{HH}$. Boxes for each treatment represent individuals falling within quartiles, as described in Figure 1; numbers within each box represent sample sizes of each treatment. 
Figure 3. Box plots of mean fitness estimates for $D$. leptopus raised under different food regimes, as described in Figure 1. Upper case letters above each boxplot identify means that are not significantly different following Tukey's HSD test.

Figure 4. Spearman rank correlations, including correlation coefficients, statistical significance and sample size, for A. Size at maturity vs. fitness in experiments 2 and 3. B. Age at maturity vs. fitness in experiments 2 and 3 , and C. Total egg production per individual female vs. fitness for experiments 2 and 3 . 

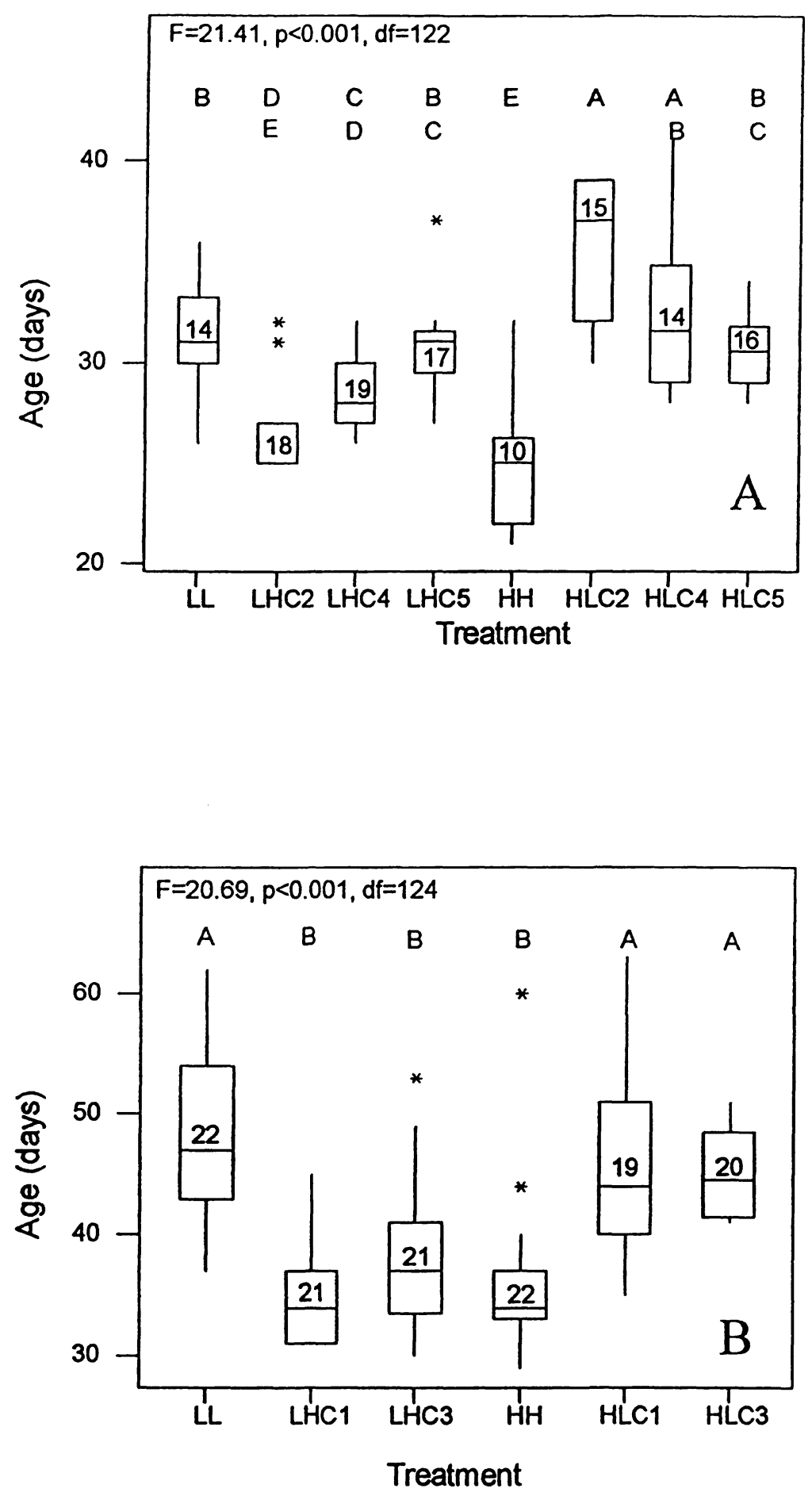
Fiquie 2

Twombly + Tisch
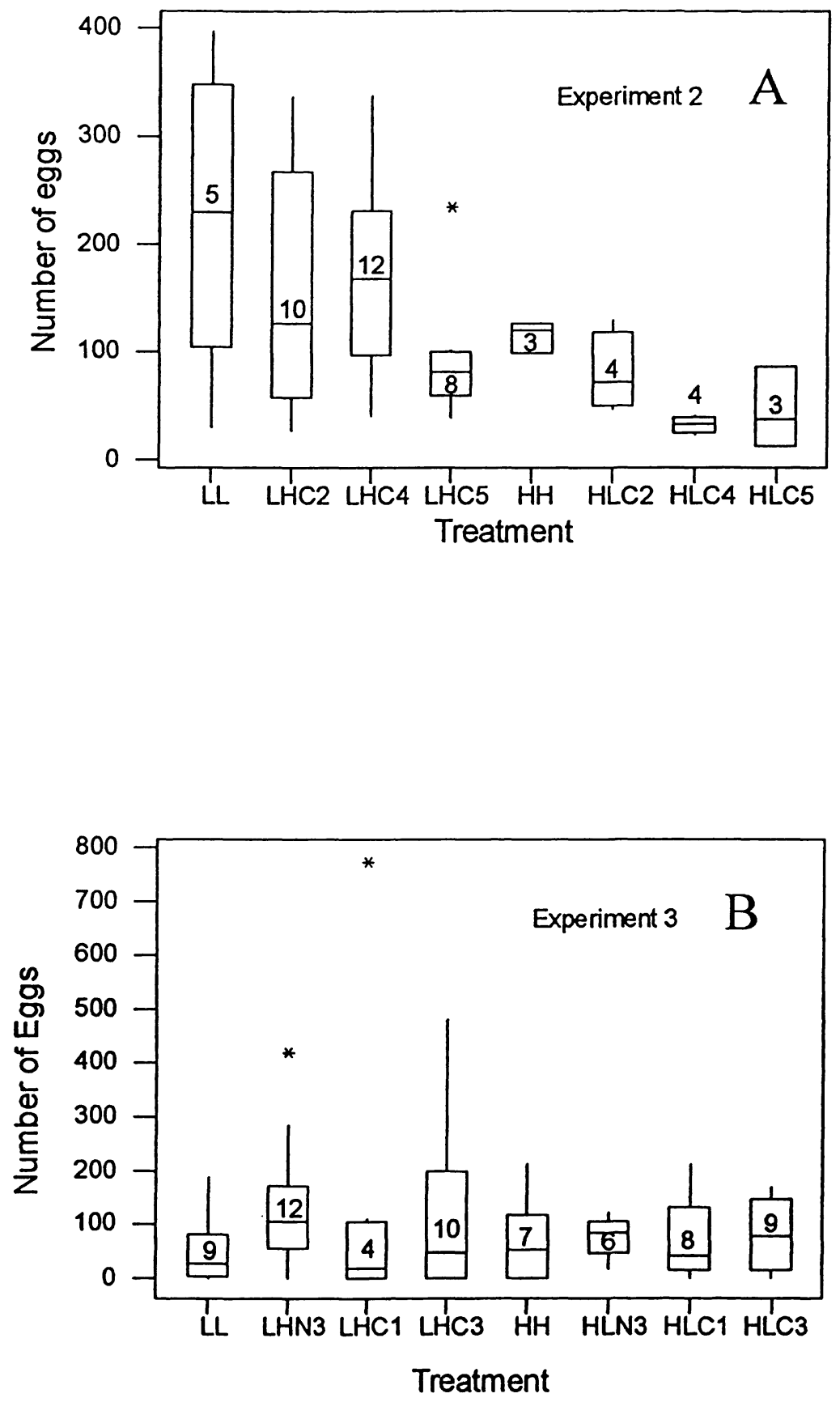
Figlie 3

Twombly + Trin
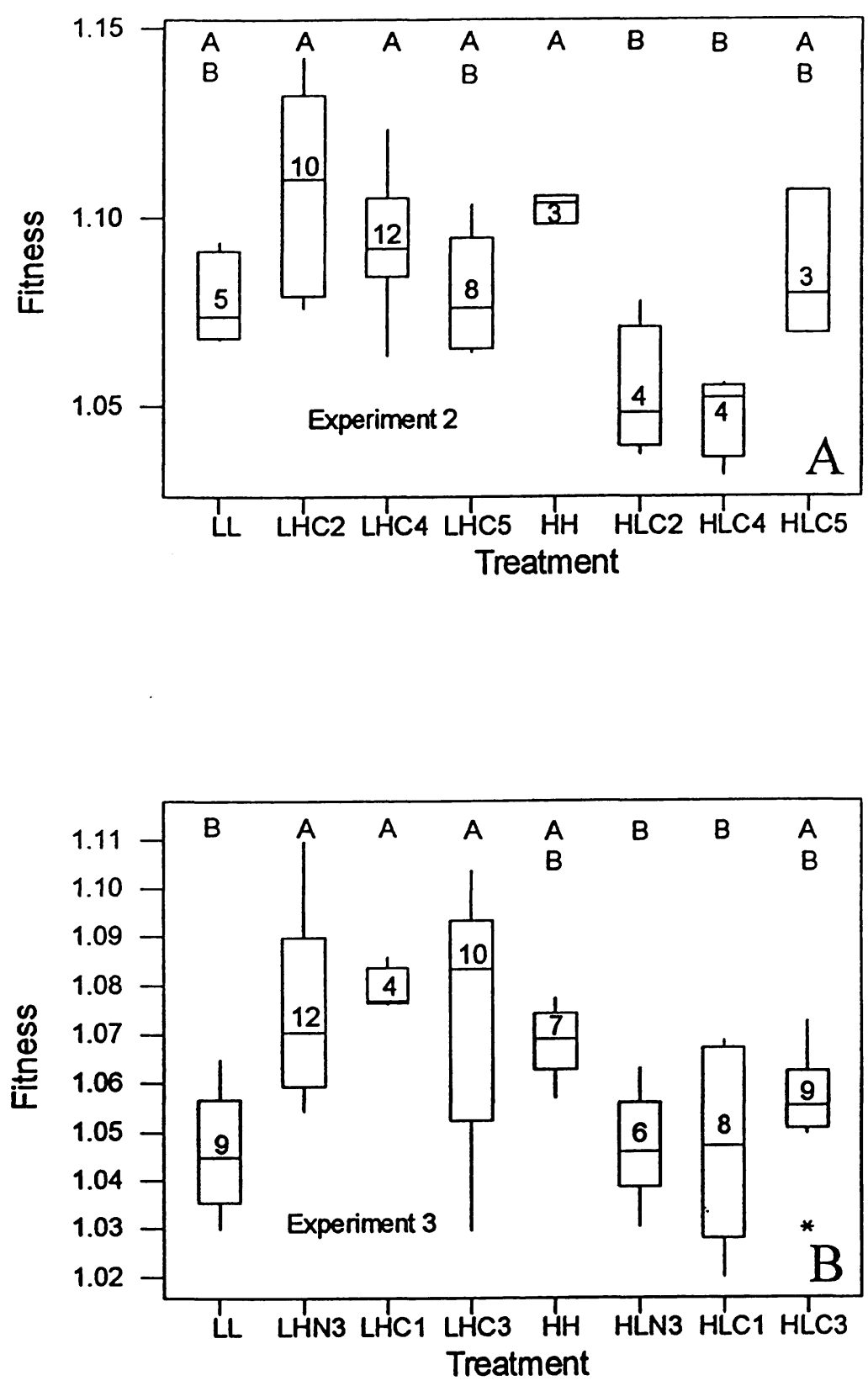
A
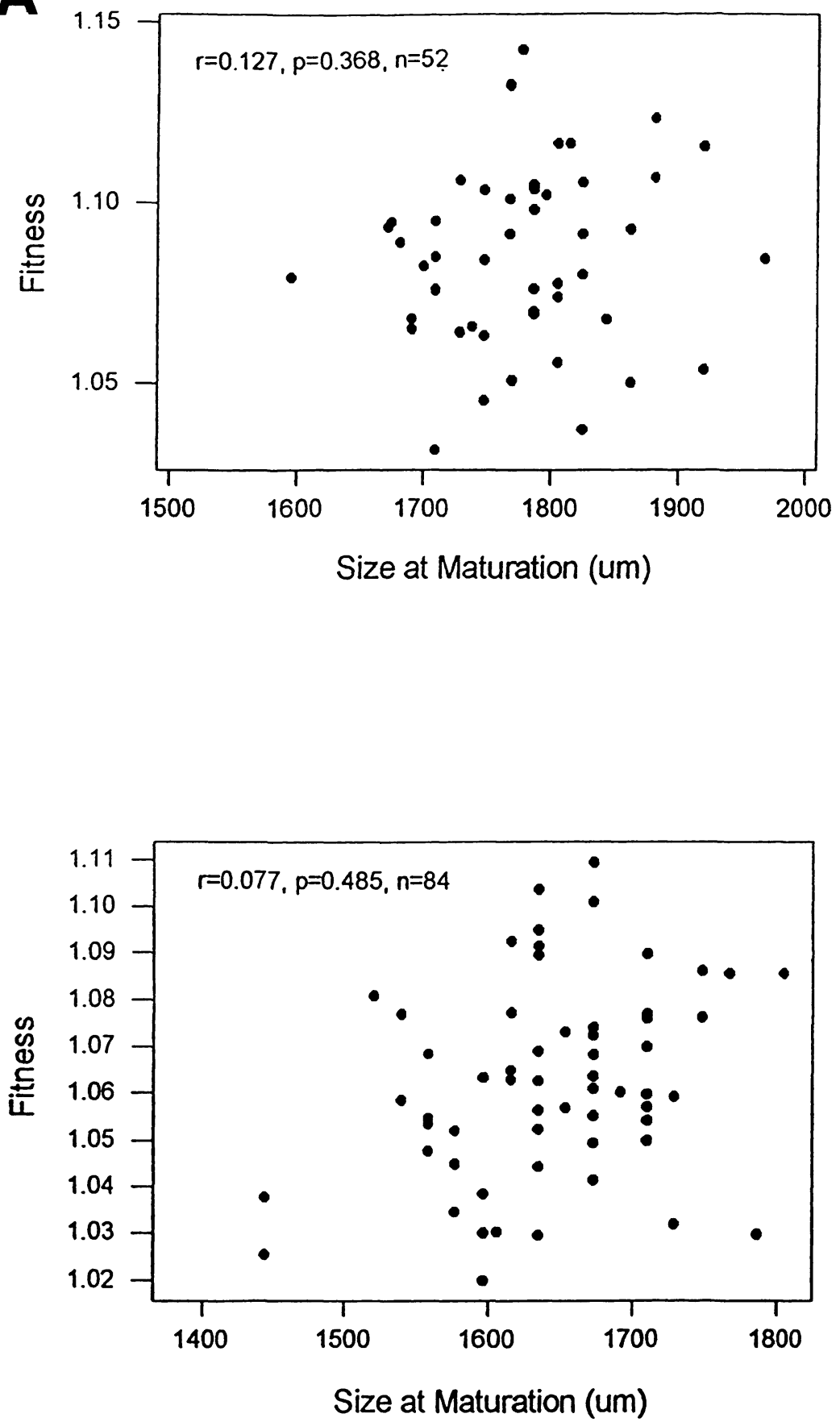

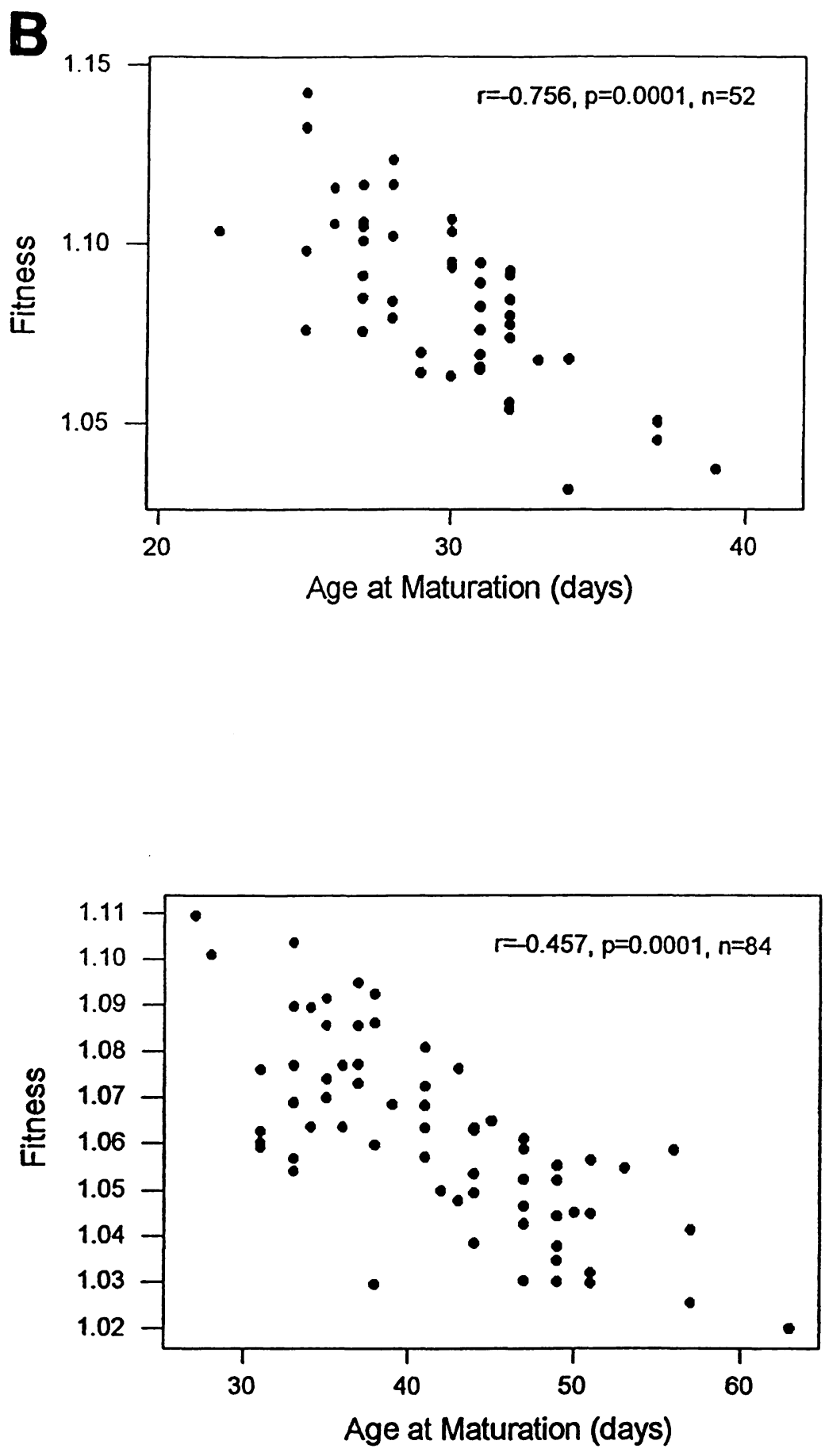

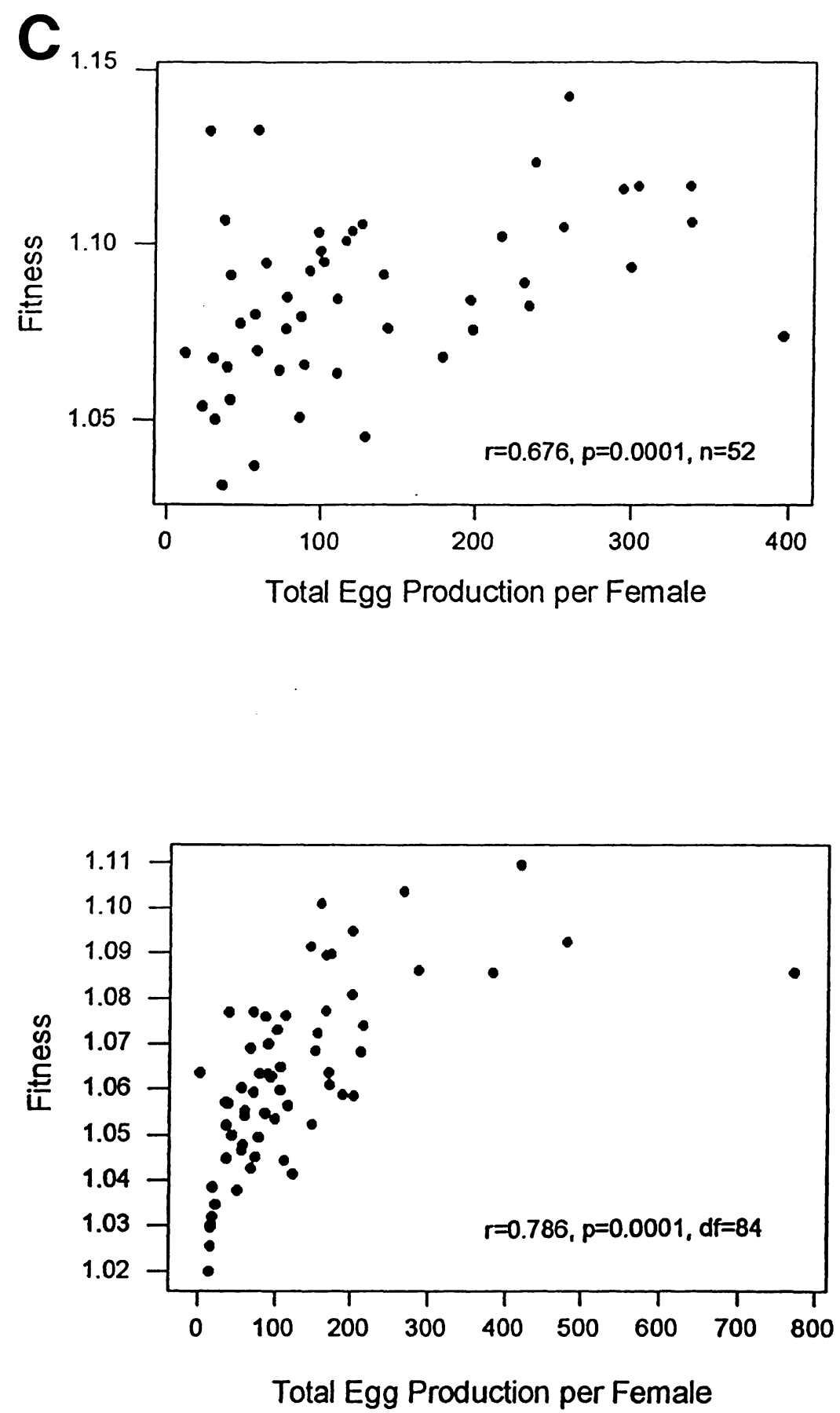